\title{
Zur Biologie des Planktons des Königshafens (Nordsylter Wattenmeer)
}

\author{
P. Martens \\ Biologische Anstalt Helgoland (Litoralstation); \\ D-2282 List/Sylt, Bundesrepublik Deutschland
}

\begin{abstract}
On the biology of the plankton of the Königshafen (northern Wadden Sea of Sylt). From May 1979 on, the following parameters were measured at a station in the inlet of Königshafen near List (Island of Sylt): temperature, salinity, mesozooplankton $(>76 \mu \mathrm{m})$, chlorophyll-a, seston dry weight, oxygen and phytoplankton-nutrients $\left(\mathrm{NH}_{4}-\mathrm{N}, \mathrm{NO}_{2}-\mathrm{N}, \mathrm{NO}_{3}-\mathrm{N}, \mathrm{PO}_{4}-\mathrm{P}, \mathrm{SiO}_{3}-\mathrm{Si}\right)$. A multiple regression analysis showed the interrelationships between the parameters measured. Tidal influences on zooplankton and seston dry weight could be observed. At low tide, the amount of zooplankton (not counting the harpacticoid copepods) declines and the number of harpacticoid copepods rises as does the seston dry weight too. The chlorophyll-a content is a function of the phytoplankton-nutrients. An increase in chlorophyll-a leads to a decrease in nitrogen and silicate concentrations. Phosphate, due possibly to a sewage inlet into the Königshafen, is not a limiting factor. The availability of nutrients is influenced by temperature, salinity and the tidal cycle. The amount of.oxygen is dependent on water temperature and seston dry weight. High water temperatures and a high seston content lead to a decrease in oxygen concentrations.
\end{abstract}

\section{EINLEITUNG}

Dem Nordsee-Wattenmeer als ökologisch einmaligem Übergangsgebiet zwischen Festland und Meer ist in den letzten Jahren verstärkt Aufmerksamkeit durch biologischozeanographische Untersuchungen zuteil geworden. Insbesondere Arbeiten des Nederlands Instituut voor Onderzoek der Zee (NIOZ), Texel, brachten einen quantitativen Aspekt in die Arbeiten auf diesem Gebiet (Cadée, 1978; Cadée \& Hegeman, 1974a, b, 1977). Die Bedeutung dieser Arbeiten wird verständlich, betrachtet man die Rolle des Wattenmeeres für den Küsten- und Naturschutz, die Landneugewinnung und insbesondere die Fischerei, für die das Wattenmeer ein wichtiges Aufzuchtgebiet für Fischbrut darstellt. Auf deutschem Gebiet ist besonders dem Nordsylter Wattenmeer seit 1975 erhöhte Aufmerksamkeit gewidmet worden (Hickel, 1975; Martens, 1980, 1981; Reise, 1978,1981 ). Ein Nachteil der bisherigen Arbeiten liegt von biologisch-ozeanographischer Sicht darin, daß ein direkter Einfluß des Wattenmeeres auf das Plankton und seine unbelebte Umwelt (Seston, Nährstoffe etc.) nur ungenügend nachzuweisen war. Dies war u. a. bedingt durch die Probennahmefrequenz und die weitgehend ungeklärten Strömungsverhältnisse, die zu einer deutlichen Inhomogenität der Wasserinhaltsstoffe im Nordsylter Wattenmeer führen (Martens, unveröff. Daten). Aus diesem Grund wurden ab 1979 weitere Untersuchungen am Königshafen vor List vorgenommen (Abb. 1).

Der Königshafen ist eine Bucht von $8 \mathrm{~km}^{2}$ Größe, die von Norden, Westen und 
Süden durch Dünen oder Marschwiesen vollständig eingeschlossen ist, so daß die Brandung der offenen See nirgends eindringen kann. Dieser Abschluß macht den inneren Teil selbst bei stärkeren Ostwinden zu einer stillen Lagune, in der sich die Vegetation ungestört entwickeln kann. Der einzige stärkere Wasserzufluß ist der zwischen Ellenbogen und der Nordspitze der Halbinsel Uthörn liegende schmale Priel (Nienburg, 1927). Der Königshafen mit maximal 1,5 m Wassertiefe bei Hochwasser fällt bei jeder Ebbe fast vollständig trocken (Kolumbe, 1933). Dies bewirkt während einer Tide starke Schwankungen im Salzgehalt und in der Temperatur. Wenn überhaupt, dann sollten Einflüsse der Tide also in diesem Gebiet feststellbar sein. Aus diesem Grund wurden vom Sommer 1979 an regelmäßig über das Jahr verteilt Untersuchungen des Wassers und seiner Inhaltsstoffe an einer Station im ein- und ausleitenden Priel jeweils über eine halbe Tide vorgenommen $(\mathrm{Abb}, 1)$.

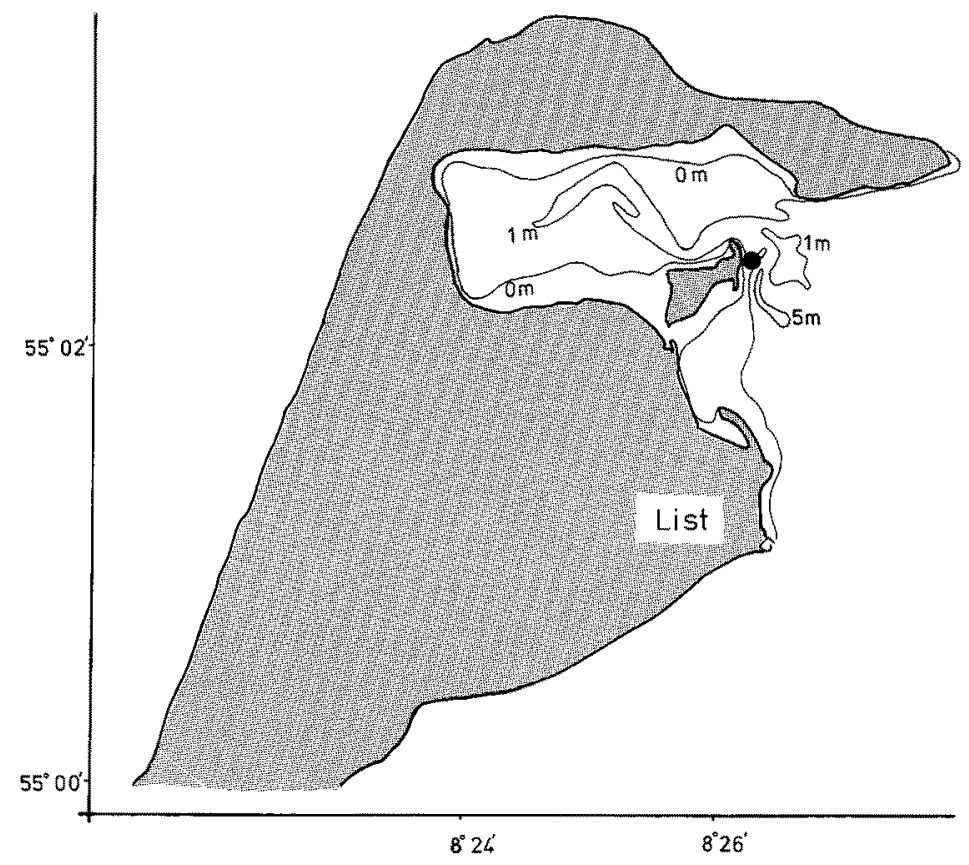

Abb. 1. Karte des nördlichen Teils der Insel Sylt mit dem Königshafen. $\bullet=$ Probennahmeort. Tiefenangaben auf Normalnull bezogen

Dieser Beitrag stellt eine Analyse der bisherigen Daten dar, um einen ersten Überblick über die Art der Abhängigkeiten zwischen den einzelnen Meßgrößen zu erhalten und die bisher angewandte Meßstrategie zu prüfen.

\section{MATERIAL UND METHODE}

Vom 28.5. 1979 an wurden gleichmäßig übers Jahr verteilt an bisher 16 Tagen jeweils über eine halbe Tide halbstündlich am einleitenden Königshafenpriel (Abb.1) folgende Parameter gemessen: (1) Temperatur ( $\pm 0,05 \mathrm{~K})$, (2) Salzgehalt $( \pm 0,003 \%$; Autolab-Salinometer), (3) Mesozooplankton (>76 $\mu \mathrm{m}$ ) (vgl. Martens, 1980), (4) Chloro- 
phyll-a (vgl. Strickland \& Parsons, 1968), (5) Sauerstoff (vgl. Grasshoff, 1976), (6) SestonTrockengewicht (vgl. v. Bröckel, 1973), (7) Phytoplankton-Nährstoffe $\left(\mathrm{PO}_{4}-\mathrm{P}, \mathrm{SiO}_{3}-\mathrm{Si}\right.$, $\mathrm{NH}_{4}-\mathrm{N}_{3} \mathrm{NO}_{2}-\mathrm{N}_{3} \mathrm{NO}_{3}-\mathrm{N}$ ) (vgl. Grasshoff, 1976).

Die Wasserproben (außer für das Mesozooplankton) wurden mit Hydro-Bios TPNWasserschöpfern $\left(3,5 \mathrm{dm}^{3}\right)$ dicht unter der Wasseroberfläche entnommen. Die geringe Wassertiefe erlaubte nur, in einer Tiefe Proben zu nehmen. Durch die starke turbulente Durchmischung des Wassers sind diese repräsentativ für die ganze Wassersäule.

Diese geringe Wassertiefe verhinderte ebenfalls den Einsatz von $10-\mathrm{dm}^{3}$-Wasserschöpfern; so mußten im Gegensatz zu früheren Untersuchungen (Martens, 1980) die Wasserproben zur Zooplanktonbestimmung mit 10-dm³ -Eimern geschöpft werden.

Nach Messungen von Hillebrandt (1972) und Martens (1976) wurden die Individuenzahlen des Mesozooplanktons in Trockengewicht umgerechnet; harpacticoide Copepoden wurden dabei gesondert erfaßt (vgl. Martens, 1980).

Aus den Zählungen des Mesozooplanktons wurde der Artendiversitätsindex nach Shannon \& Weaver (Parsons \& Takahashi, 1977) berechnet nach der Formel $H=-\Sigma p_{i} \times \log _{2} p_{i}$ wobei $p_{i}=\mathrm{n}_{\mathrm{i}} / \mathrm{N}$ mit $\mathrm{n}_{\mathrm{i}}=$ Anzahl der Tiere der i-ten Art und $\mathrm{N}=$ Gesamtindividuenzahl.

Im Zuge der statistischen Analyse wurden $\mathrm{NH}_{4}-\mathrm{N}, \mathrm{NO}_{2}-\mathrm{N}$ und $\mathrm{NO}_{3}-\mathrm{N}$ als "Gesamtstickstoff"' ( $\Sigma$ Stickstoff) behandelt.

\section{STATISTISCHE BERECHNUNGEN}

Folgende Parameter wurden als Zielgrößen gewählt (Sachs, 1974): Seston-TG, Chlorophyll-a, $\Sigma$ Stickstoff, Phosphat, Silikat, Sauerstoff, Zooplankton-TG (ohne Harpacticiden), Harpacticiden und der Artendiversitätsindex.

Als Einflußgrößen (Sachs, 1974) wurden für jede Zielgröße außer den oben genannten Meßgrößen noch die Tide gewählt (in Prozent vom Hochwasser; Niedrigwasser = $0 \%$, Hochwasser $=100 \%$ ).

Das Maß der formalen Abhängigkeit der einzelnen Zielgrößen von jeder einzelnen Einflußgröße wurde durch Berechnung des Korrelationskoeffizienten $r$ bestimmt.

Beí dieser Vielzahl von Variablen kann in vielen Fällen davon ausgegangen werden, daß eine Zielgröße mit mehreren Einflußgrößen korreliert ist. Die formale Abhängigkeit wird dabei gegeben durch den multiplen Korrelationskoeffizienten $R$ (Sachs, 1974). Nicht jede Einflußgröße hat jedoch einen signifikanten Einfluß auf jede der angegebenen Zielgrößen. Durch Berechnung der Prüfgröße $\hat{F}$ (Sachs, 1974) wird untersucht, ob durch die Hinzunahme einer weiteren Einflußgröße ein signifikant höherer multipler Korrelationskoeffizient erreicht wird. Somit wird für jede der genannten Zielgrößen das Maß der formalen statistischen Abhängigkeit von einer oder auch mehreren Einflußgrößen bestimmt, wobei die Hinzunahme weiterer Variabler keine wesentliche Verbesserung ergäbe. Die Art der formalen Abhängigkeit wird im weiteren bestimmt durch Berechnung der multiplen linearen Regression (Sachs, 1974). Eine Signifikanz der Regressionskoeffizienten ergibt sich aus der Tatsache, daß die Vorhersage der Zielgröße durch Hinzunahme der entsprechenden Variablen signifikant verbessert wurde.

Voraussetzung zur Berechtigung der Anwendung dieser statistischen Methoden ist eine, wenigstens angenäherte Normalverteilung der beteiligten Größen. Dies ist jedoch 
gerade bei biologischen Untersuchungen äußerst selten gegeben. Der häufig gemachte Vorschlag, auf die Rang-Korrelation auszuweichen, führt jedoch lediglich zu qualitativen Aussagen und erlaubt keine weitergehenden über komplexe Zusammenhänge. Wenn also trotz der oben genannten Einwände die statistischen Berechnungen in der hier angewandten Form durchgeführt wurden, muß man sich der Einschränkung der Sicherheit der Aussage bewußt sein. Es gilt hier also noch mehr auf die biologische Sinnhaftigkeit der formalen statistischen Aussage zu achten. Trifft diese zusammen mit einer möglichst hoch zu wählenden formalen Sicherung, wird die hier angewandte Methode für vertretbar gehalten.

\section{ERGEBNISSE UND DISKUSSION}

Die Auswertung zeigt, daß der Sestongehalt des Wassers hinreichend genau durch Temperatur, Salzgehalt und Tide zu beschreiben ist (Tab. 1). Bei Niedrigwasser ist ein höherer Sestongehalt zu erwarten als bei Hochwasser. Der Sestongehalt stellt also unter anderem eine Funktion der Wassertiefe dar.

Der Chlorophyll-a-Gehalt des Wassers ist positiv korreliert mit dem PhosphatGehalt und negativ korreliert mit dem Silikat- und Stickstoffgehalt. Das übliche Schema der Nährstoffabnahme im Frühjahr bei zunehmendem Phytoplankton bis zum allmählichen Wiederanstieg des Nährstoffgehaltes im Herbst-Winter bei allmählich abnehmender Phytoplanktonmenge trifft hier also nur auf Silikat und Stickstoff $\mathrm{zu}$, nicht auf Phosphat, das zudem eine deutliche Tidenabhängigkeit mit Zunahme bei Niedrigwasser zeigt (Tab. 1). Dies könnte bedingt sein durch die Einleitung von Abwässern einer nahe gelegenen Jugendherberge in den Königshafen. Nach Auskunft der Jugendherbergsleitung handelt es sich vorwiegend um phosphatreiches Waschwasser, das ohne größere jahreszeitliche Schwankungen anfällt.

Bei allen drei Nährstoffen ist eine Verarmung bei zunehmendem Salzgehalt zu beobachten (Tab. 1). Einströmendes Nordseewasser, das gegenüber dem Niedrigwasser einen höheren Salzgehalt aufweist (Vorzeichentest; $\alpha<2,5 \%$, vgl. Lienert, 1973), hat demnach einen geringeren Nährstoffgehalt. Eine Erklärung wäre die hohe Remineralisation auf den flachen Wattengebieten mit ihren hohen biologischen Umsatzraten. Insbesondere trifft dies zu bei höheren Temperaturen, wie eine positive Korrelation von Phosphat und Silikat mit der Wassertemperatur zeigt.

Bei dem Parameter $\Sigma$ Stickstoff ist dies anders. Die einzelnen Komponenten haben unterschiedliche Arten der Temperaturabhängigkeit, die im Rahmen dieser Arbeit nicht weiter untersucht werden sollen. Der Sauerstoffgehalt des Wassers ist negativ korreliert mit der Temperatur und dem Sestongehalt - bei höheren Temperaturen ist physikalisch bedingt weniger Sauerstoff im Wasser gelöst. Ein höherer Sestongehalt führt zu einer Sauerstoffabnahme durch bakterielle aerobe Aktivitäten bei Zersetzung organischer Substanzen.

Ebenso deutlich ist das Bild bei den harpacticoiden Copepoden. Höhere Temperaturen bewirken eine Bestandszunahme durch die Initiierung der Eisackbildung (Mielke, 1976). Auch in früheren Untersuchungen ist eine deutliche Parallelentwicklung von Harpacticidenbestand und Wassertemperatur beobachtet worden (Arlt, 1973; Scheibel, 1973). Je niedriger der Wasserstand ist, desto höher ist die Anzahl der Harpacticiden. Ihr Vorkommen ist also primär im Watt zu suchen (Abb. 2). 


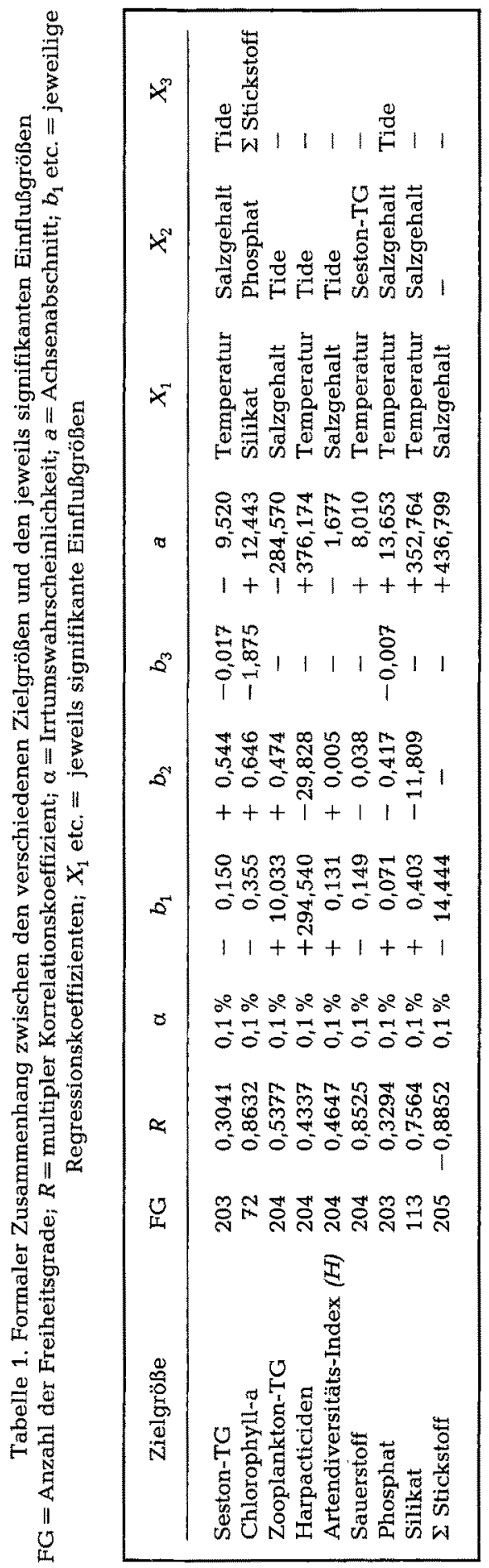




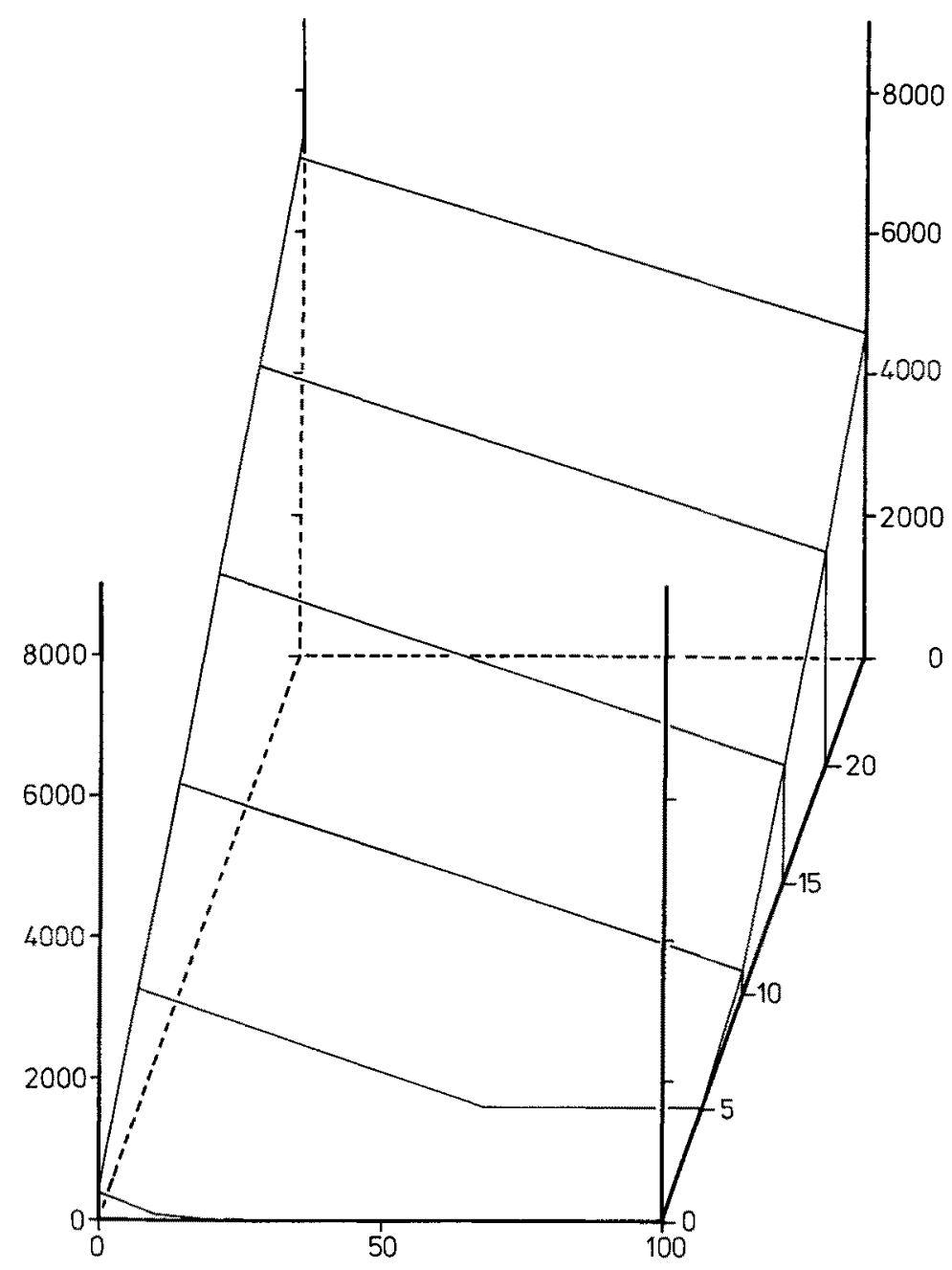

Abb. 2. Abhängigkeit der Anzahl der Harpacticiden pro $\mathrm{m}^{3}$ ( $\mathrm{Y}$-Achse) von der Wassertemperatur (Z-Achse) und dem Tidenstand (X-Achse) $(0 \%=$ Niedrigwasser; $100 \%=$ Hochwasser). Harpacticiden $=376,2+294,5 \times$ Temperatur $-29,8 \times$ Tide. Korrelationskoeffizient $R=0,434$; Anzahl der Freiheitsgrade $=204 ;$ Irrtumswahrscheinlichkeit $\alpha<0,1 \%$

Das restliche Zooplankton verhält sich nicht in dieser Form. Eine Temperaturabhängigkeit ist nicht zu erkennen. Wie bei $\Sigma$ Stickstoff handelt es sich um einen Summenparameter vieler unterschiedlicher Komponenten, die verschiedene Temperaturoptima haben. Eine positive Korrelation besteht mit dem Salzgehalt. Einströmendes salzreiches Nordseewasser führt Zooplankton mit sich, das zum Niedrigwasser deutlich abnimmt, wie eine negative Korrelation mit der Tide anzeigt (Abb. 3).

Dieser negative Einfluß des Wattgebietes auf das Zooplankton ist auch im Artendiversitätsindex zu sehen. Eine Abnahme des Index ist deutlich mit einer Abnahme der Tide korreliert. 


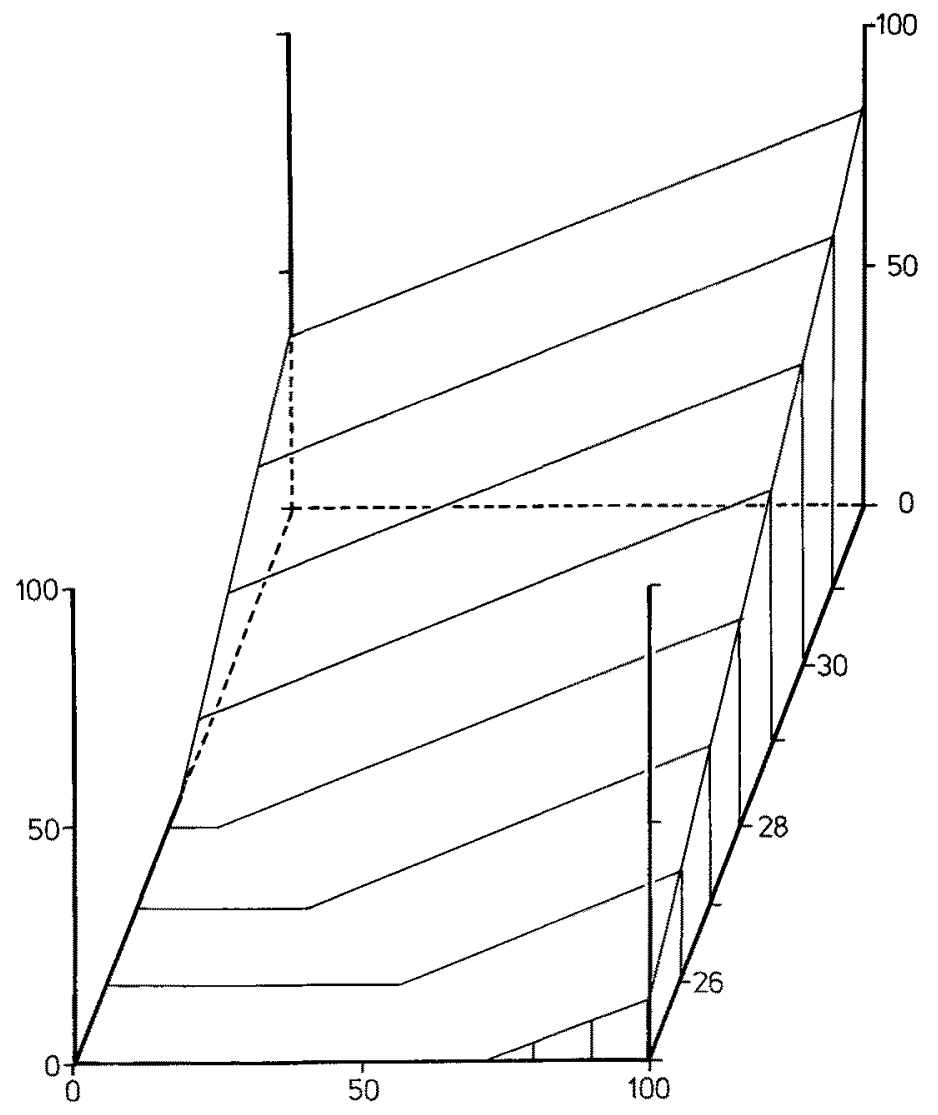

Abb. 3. Abhängigkeit der Menge des Zooplanktons in mg Trockengewicht pro $\mathrm{m}^{3}$ (ohne Harpacticiden) (Y-Achse) von dem Salzgehalt in \% (Z-Achse) und dem Tidenstand (X-Achse) $10 \%=$ Niedrigwasser; $100 \%=$ Hochwasser). Zooplankton-Trockengewicht $=-284,57+10,03 \times$ Salzgehalt $+0,47 \times$ Tide. Korrelationskoeffizient $R=0,538$; Anzahl der Freiheitsgrade $=204$; Irrtumswahrscheinlichkeit $\alpha<0,1 \%$

Bedenkt man, daß es sich bei dem Niedrigwasser um das von den flachen Wattengebieten des Königshafens ablaufende Wasser handelt, ist ein deutlicher Einfluß des Watts auf die Wassersäule feststellbar. Einströmende Zooplanktonpopulationen sind einem starken Streß ausgesetzt, der sowohl zu einer Artenverarmung als auch zu einer Bestandsdezimierung führt. Das Zooplankton der offenen Nordsee wird ersetzt durch die Harpacticidenpopulationen des Wattgebietes. Gleichzeitig steigt der Sestongehalt des Wassers, und, besonders bei höheren Temperaturen, die Menge der Nährstoffe.

Welcher Art der negative Einfluß des Watts auf das Zooplankton ist, konnte bei dieser Meßstrategie nicht ermittelt werden. Möglich wäre zum Beispiel eine Zehrung durch planktivore Fischbrut, die im Wattgebiet in großen Bestandsdichten gefunden werden kann (Zijlstra, 1977), oder durch die ausgedehnten Muschelbänke. Auch ein Streß durch den sehr hohen Detritus-Gehalt des Wassers wäre denkbar. Mit seinem 
großen Anteil an mineralischen Substanzen (Hickel, unveröff. Daten) stellt er eine schwer verwertbare Nahrung dar, sofern die Zooplankter nicht zu selektiver Nahrungsaufnahme fähig sind, was von Acartia clausi bekannt ist (Poulet \& Marsot, 1978). Dies ist eine Möglichkeit zur Erklärung ihres erhöhten Auftretens im Wattgebiet gegenüber anderen Copepodenarten (Martens, 1981).

Bei der weiteren Durchführung dieser Untersuchungen, die mehrjährig geplant sind, sollte also die Zusammenarbeit mit Fischereibiologen angestrebt werden, um den Einfluß der Jungfische auf das Zooplankton zu quantifizieren, sowie mit Zoobenthologen zur Erfassung des Einflusses der Muschelbänke. Ferner sollte in Laborexperimenten, die bereits angelaufen sind (Weisse, 1982), weiter an der Frage der Nahrungsbiologie des Zooplanktons gearbeitet werden.

Die Meßstrategie, die bisher angewandt wurde, erscheint zufriedenstellend. Die wechselseitige Abhängigkeit der verschiedenen Parameter ließ sich formal deutlich nachweisen. Die Untersuchungen sollen daher in dem hier dargestellten Rahmen langfristig fortgeführt werden.

Danksagung. Frau C. Reineke danke ich für die Durchführung der chemischen Analysen, der Planktonzählungen und der Vorbereitung und Durchfürung der Ausfahrten mit dem Forschungskatamaran "MYA", dessen Besatzung uns eine große Hilfe war.

\section{ZITIERTE LITERATUR}

Arit, G., 1973. Jahreszeitliche Fluktuationen der Meiofauna im Greifswalder Bodden. - Wiss. Z. Univ, Rostock 22, 685-692.

Bröckel, K. v., 1973. Eine Methode zur Bestimmung des Kaloriengehaltes von Seston. - Kieler Meeresforsch. 29, 34-49.

Cadée, G., 1978. On the origin of organic matter accumulating on the tidal flats of Balgzand, Dutch Wadden Sea. - Hydrobiol. Bull. 12, 145-150.

Cadée, G. \& Hegeman, J., 1974a. Primary production of the benthic microflora living on tidal flats in the Dutch Wadden Sea. - Neth. J. Sea Res. 8, 260-291.

Cadée, G. \& Hegeman, J., 1974b. Primary production of phytoplankton in the Dutch Wadden Sea.Neth. J. Sea Res. 8, 240-259.

Cadée, G. \& Hegeman, J., 1977. Distribution of primary production of the benthic microflora and accumulation of organic matter on a tidal flat area, Balgzand, Dutch Wadden Sea. - Neth. J. Sea Res. $11,24-41$.

Grasshoff, $K_{*}$ 1976. Methods of seawater analysis. Verl. Chemie, Weinheim, $297 \mathrm{pp}$.

Hickel, W, 1975. The mesozooplankton of the Wadden Sea of Sylt (North Sea). - Helgoländer wiss. Meeresunters. 27, 254-262.

Hillebrandt, M., 1972. Untersuchungen über die qualitative und quantitative Zusammensetzung des Zooplankton in der Kieler Bucht während der Jahre 1966-1968. Diss., Univ. Kiel, 138 pp.

Kolumbe, E, 1933. Ein Beitrag zur Kenntnis der Entwicklungsgeschichte des Königshafens bei List auf Sylt. - Wiss. Meeresunters. (Abt. Kiel) 21, 117-130.

Lienert, G. A., 1973. Verteilungsfreie Methoden in der Biostatistik. Hain, Meisenheim am Glan, 1, $1-736$.

Martens, P., 1976. Die planktischen Sekundär- und Tertiärproduzenten im Flachwasserökosystem der westlichen Ostsee. - Kieler Meeresforsch. (Sonderh.) 3, 60-71.

Martens, P., 1980. Beiträge zum Mesozooplankton des Nordsylter Wattenmeers. - Helgoländer Meeresunters. 34, 41-53.

Martens, P., 1981. On the Acartia species of the northern Wadden Sea of Sylt. - Kieler Meeresforsch. (Sonderh.) 5, 153-163. 
Mielke, W., 1976. Okologie der Copepoda eines Sandstrandes der Nordseeinsel Sylt. - Mikrofauna Meeresboden 59, 453-536.

Nienburg, W., 1927. Zur Okologie der Flora des Wattenmeeres 1. Teil: Der Königshafen bei List auf Sylt. - Wiss. Meeresunters. (Abt. Kiel) 20, 149-195.

Parsons, T. R. \& Takahashi, M., 1977. Biological oceanographic processes. Pergamon Press, Oxford, $332 \mathrm{pp}$.

Poulet, S. A. \& Marsot, P, 1978. Chemosensory grazing by marine calanoid copepods (Arthropoda: Crustarea). - Science, N.Y. 200, 1403-1405.

Reise, K., 1978. Experiments on epibenthic predation in the Wadden Sea. - Felgoländer wiss. Meeresunters. 31, 55-101.

Reise, K., 1981. High abundance of small zoobenthos around biogenic structures in tidal sediments of the Wadden Sea. - Helgoländer Meeresunters. 34, 413-425.

Sachs, L., 1974. Angewandte Statistik. Springer, Berlin, 545 pp.

Scheibel, W., 1973. Quantitativ-ökologische Untersuchungen am uferfernen Mesopsammon in der Kieler Bucht. - Kieler Meeresforsch. 29, 58-68.

Strickland, J. D. H. \& Parsons, T. R., 1968. A practical handbook of seawater analysis. - Bull. Fish. Res. Bd Can, 167, 1-310.

Weisse, T., 1982. Über die Auswirkungen einer Phaeocystis pouchetii-Blüte auf die dominierenden Copepodenarten des Nordsylter Wattenmeeres. Dipl-Arbeit, Univ. Kiel.

Zijlstra, J., 1977. Fische. In: Wattenmeer, Hrsg. von J. Abrahamse, W. Joenje \& N. v. Leeuwen-Selt. Wachholz, Neumünster, $372 \mathrm{pp}$. 